\section{Photoacoustic imaging of the microvasculature with a high-frequency ultrasound array transducer}

\author{
Roger J. Zemp, ${ }^{a}$ Rachel Bitton, ${ }^{b}$ Meng-Lin Li, \\ K. Kirk Shung, ${ }^{b}$ George Stoica, ${ }^{d}$ and Lihong V. Wang ${ }^{\mathrm{e}, *}$ \\ ${ }^{a}$ Washington University, Optical Imaging Laboratory, \\ Department of Biomedical Engineering, Saint Louis, Missouri \\ 63130 \\ ${ }^{b}$ University of Southern California, Department of \\ Biomedical Engineering, Los Angeles, California 90089 \\ ${ }^{\mathrm{C}}$ National Tsing Hua University, Department of Electrical \\ Engineering, Hsinchu 30013, Taiwan \\ ${ }^{\mathrm{d}}$ Texas A\&M University, Department of Veterinary \\ Pathobiology, College Station, Texas 77843 \\ 'Washington University, Optical Imaging Laboratory, \\ Departments of Biomedical Engineering and Radiology, Saint \\ Louis, Missouri 63130
}

\begin{abstract}
Visualization of microvascular networks could provide new information about function and disease. We demonstrate the capabilities of a $30-\mathrm{MHz}$ ultrasound array system for photoacoustic microscopy of small $(\leq 300 \mu \mathrm{m})$ vessels in a rat. 3D images obtained by translating the array in the elevation direction are compared with photographs of excised skin. The system is shown to have $100-\mu \mathrm{m}$ lateral resolution, $25-\mu \mathrm{m}$ axial resolution, and 3-mm imaging depth. To our knowledge this is the first report on photoacoustic microscopy of the microvasculature with a high-frequency array transducer. It is anticipated that the system can be used for studying and diagnosing a number of diseases including cancer, atherosclerosis, dermatological disorders, and peripheral microvascular complications in diabetes. () 2007 Society of PhotoOptical Instrumentation Engineers. [DOI: 10.1117/1.2709850]
\end{abstract}

Keywords: microcirculation; microvascular; photoacoustic imaging; photoacoustic microscopy; biophotonic imaging; optical absorption; high-frequency array transducer; high-frequency ultrasound; ultrasound arrays.

Paper 06299LRR received Oct. 24, 2006; revised manuscript received Dec. 21, 2006; accepted for publication Dec. 22, 2006; published online Mar. 1, 2007.

The microvasculature plays an important role in tissue function and pathogenesis, ${ }^{1}$ yet in many diseases the etiology of microvascular dysfunction is poorly understood. There is a significant need for technologies to visualize microvascular growth and remodeling in the pathogenic process. Early detection of abnormal microvascular dynamics and morphology could prove important in effective interventional strategies. Additionally, microvascular imaging technologies may provide a much needed tool for basic research and for monitoring therapeutic efficacy in vivo.

\footnotetext{
*E-mail: Ihwang@biomed.wustl.edu
}

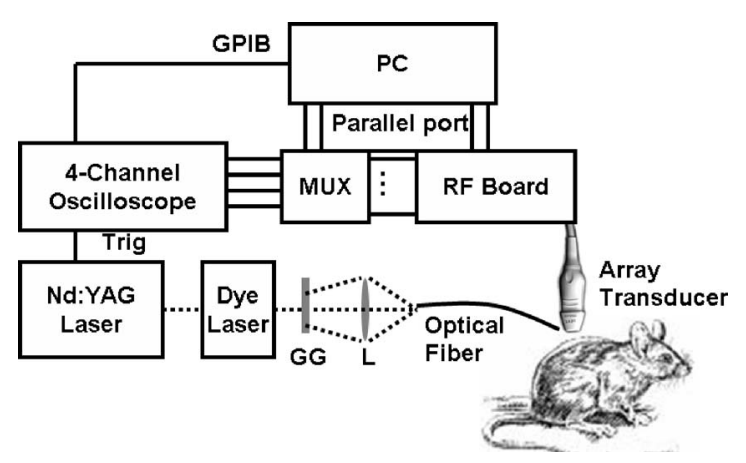

Fig. 1 System diagram. GG: ground-glass diffuser; L: lens; MUX: multiplexer.

Photoacoustic imaging has the potential to address some of these needs. In photoacoustic imaging, short pulses of laser light are transmitted into the body. Upon light absorption, a rapid thermoelastic expansion launches acoustic waves that are detected and reconstructed to form images representing optical absorption. Since blood has a visible-light absorption coefficient one to two orders of magnitude larger than surrounding tissue, there is significant endogenous contrast available for imaging blood vessels. Recently, our group reported a novel scanning photoacoustic microscopy system with darkfield illumination and a high numerical aperture $50-\mathrm{MHz}$ single-element ultrasound transducer. ${ }^{2,3}$ This system produces clear images of microvessels to depths of about $3 \mathrm{~mm}$; however, it requires mechanical scanning and has only 1-mm depth of field.

We present a photoacoustic microscopy system with a 30 $\mathrm{MHz}$ ultrasound array transducer. Advantages of this array over a mechanically scanned single element transducer include the ability to use dynamic receive focusing (greatly increasing the depth of field), electronic rather than mechanical B-scanning capability, and parallel data acquisition. Eventually the probe may be handheld and may provide real-time imaging capabilities. Other groups are beginning to use arrays for photoacoustic imaging, ${ }^{4}$ but this is the first report of highfrequency (above $20 \mathrm{MHz}$ ) photoacoustic imaging of the microvasculature. Due to small detection areas of individual elements, there may be some question regarding whether highfrequency arrays are sensitive enough for imaging in vivo. We show feasibility of the high-frequency array system for photoacoustic visualization of microvessels to depths of $3 \mathrm{~mm}$ in rats in situ. Due to multiply scattered light, this depth is not attainable by present optical microscopy techniques such as confocal and two-photon microscopy, and is less invasive than intravital techniques. It is anticipated that systems similar to the one reported here may be used for studying and diagnosing a number of diseases where microvascular changes impact health, including cancer, ${ }^{1}$ dermatological disorders, cardiovascular disease, and peripheral microvascular complications in diabetics. High-frequency photoacoustic imaging may complement high-frequency Doppler ultrasound ${ }^{5,6}$ to detect very small vessels as well as those with low flow velocities.

Our system, shown in Fig. 1, consists of an ultrasound

1083-3668/2007/12(1)/010501/3/\$25.00 @ 2007 SPIE 

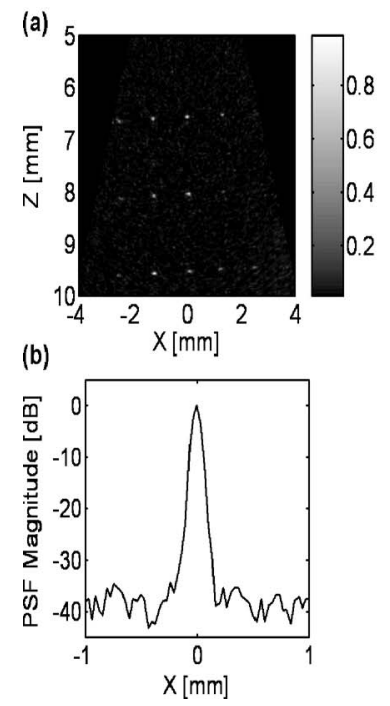

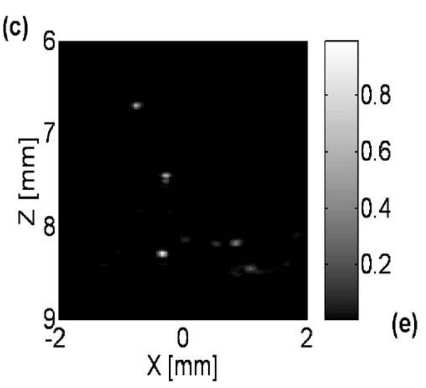

(d)

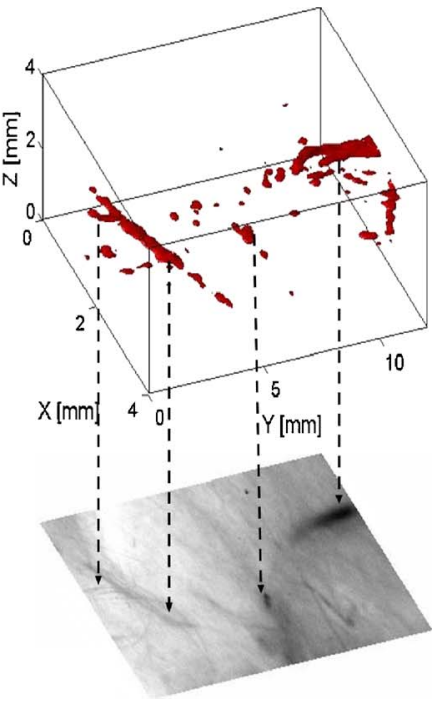

Fig. 2 (a) Composite B-scan of $6 \mu \mathrm{m}$ carbon fibers in water. (b) B-scan of a single 6 - $\mu \mathrm{m}$ carbon fiber in water, projected on the $x$ axis. (c) Photoacoustic B-scan of subcutaneous microvessels in a young rat. In (a) and (c) the $z$ axis is depth from the transducer surface in millimeters. (d) Surface-rendered photoacoustic image of volumetric data acquired by translating the array in the $y$ direction. The $z$ axis is depth from the skin surface in millimeters, with the skin surface at the bottom of the plot. (e) Photograph of the underside of excised skin in the area of photoacoustic imaging.

array, a laser system, and receiving electronics. The ultrasound transducer is a $30-\mathrm{MHz}$ piezo-composite array of 48 elements ${ }^{7}$ with $50 \%$ pulse-echo -6 - $\mathrm{dB}$ fractional bandwidth, $100-\mu \mathrm{m}$ pitch (2 times the center wavelength), 2-mm elevation aperture and 8.2-mm elevational focus, and 19.1-dB insertion loss (41.2-dB uncompensated loss, with 18.37-, 0.6-, and 3.17-dB losses due to diffraction, reflector absorption, and attenuation, respectively). These pulse-echo measurements were tested with a steel reflector placed at the focal position in water. Depending on fabrication technology, comparable frequency single element transducers have been measured to have insertion losses of 20 to $50 \mathrm{~dB}{ }^{8}$ Signals from the array were amplified by a custom rf board with $33-\mathrm{dB}$ fixed gain and an additional $40 \mathrm{~dB}$ of variable gain. The board multiplexed the 48 channels down to 16 . Four 4-to- $1 \mathrm{rf}$ multiplexer boards (MAX4141) were then used to enable data acquisition with a four-channel oscilloscope (Tektronix, TDS5034). A PC running Labview controlled multiplexing via the parallel port, while also communicating with the oscilloscope via a general purpose interface bus (GPIB). The maximum data acquisition rate was limited by the $10-\mathrm{Hz}$ pulse-repetition rate of the laser system. Acquired data were processed offline.

A Q-switched Nd:YAG laser (Quantel, Brilliant B) was used to pump a dye laser (Continuum ND6000) to obtain 6-ns pulses of wavelength $584 \mathrm{~nm}$, an isosbestic point. Laser pulses were coupled into a $0.37 \mathrm{NA}, 600-\mu \mathrm{m}$ optical fiber using a fiber coupler consisting of a ground-glass diffuser, then a lens to direct a fraction of the diffused light directly into the core of the fiber. Approximately $0.3 \mathrm{~mJ}$ of light was delivered through the fiber, which was positioned obliquely at the side of the array, forming an elliptical illumination pattern $(\sim 6 \times 3 \mathrm{~mm})$ with the major axis parallel to the array scanning plane. Approximately $6 \mathrm{~mJ} / \mathrm{cm}^{2}$ of light was delivered to the skin surface, approximately $30 \%$ of the permissible ANSI visible light exposure limit of $20 \mathrm{~mJ} / \mathrm{cm}^{2}$ per pulse.
Channel data were acquired at $250 \mathrm{MS} / \mathrm{s}$ and processed using a sector-scan beam-forming algorithm modified from ultrasound beam forming. To reconstruct an A-scan line along a line of sight defined by the angle $\theta$, we apply dynamic delays $\tau_{n}(R, \theta)$ to the received channel data $S(n, t), n$ $=1,2, \ldots, 48$, where $R$ is the distance from the image point to the beam-forming origin (taken here as the center of the array), then add the delayed data. The delays are given as

$$
\tau_{n}(R, \theta)=-\frac{x_{n} \sin \theta}{c}+\frac{x_{n}^{2} \cos ^{2} \theta}{2 R c},
$$

where $x_{n}$ is the distance to the array element from the array center, and $c$ is the speed of sound. This expression is a second-order Taylor approximation in $x_{n}$ to the transit time

$$
\left[\left(x_{n}-R \sin \theta\right)^{2}+R^{2} \cos ^{2} \theta\right] / c
$$

between a field point and element $x_{n}$. The first term of Eq. (1) represents steering, while the second represents focusing. The transit time between a point of interest and the array for pulseecho ultrasound is $t=2 R / c$, while in photoacoustic imaging propagation is one way, and $t=R / c$. Hence the steering term $-x_{n} \sin \theta / c$ is identical to pulse-echo ultrasound, while the focusing term $x_{n}^{2} \cos ^{2} \theta / 2 c^{2} t$ is different. In beam forming, we used all 48 elements to leverage the maximum possible aperture. We also found it advantageous to apply a coherence factor $(\mathrm{CF})$ weighting in the beam-forming procedure. ${ }^{9} \mathrm{~A}$ coherence factor for an A-scan line with direction $\theta_{m}$ is given as

$$
C F\left(t, \theta_{m}\right)=\frac{\left|\sum_{n=0}^{N-1} S\left[n, t-\tau_{n}\left(R, \theta_{m}\right)\right]\right|^{2}}{N \sum_{n=0}^{N-1}\left|S\left[n, t-\tau_{n}\left(R, \theta_{m}\right)\right]\right|^{2}},
$$

where $N$ is the number of elements. This time-varying function is applied multiplicatively to each beam-formed A-scan 
and tends to emphasize highly in-phase signals while rejecting low coherence side lobes, grating lobes, and noise.

To demonstrate the spatial resolution and dynamic focusing capability of the system, we imaged 6 - $\mu$ m-diam carbon fibers in water at various spatial locations to produce a composite image, shown in Fig. 2(a). Importantly, the pointspread functions are well focused throughout the region of interest. Figure 2(b) shows the projection of a single pointspread function on the $x$ axis. The $-6-\mathrm{dB}$ axial and lateral spatial resolution for points near the elevation focus were measured as 25 and $100 \pm 5 \mu \mathrm{m}$, respectively. Clutter and noise levels (taken as the mean level of the envelope-detected signal relative to the peak) were approximately $-33 \mathrm{~dB}$ when no coherence-factor weighting was used in beam forming. When coherence-factor weighting was applied, mean clutter and noise levels reduced to $-60 \mathrm{~dB}$.

To demonstrate the ability to see microvessels in animals, we imaged a young Sprague Dawley rat approximately $100 \mathrm{~g}$ in weight. The hair was removed from the back right side of the rat while the rat was anesthetized, and the animal was sacrificed prior to imaging to reduce motion artifacts. All animal procedures were performed in accordance with approved protocols.

The array and optical fibers were mounted on a 3-axis translation stage, and lowered into a water tank with an acoustically and optically transparent aperture for the animal below the tank. Figure 2(c) shows a photoacoustic B-scan image at one imaging location with several small vessels visible at 2to 3-mm depths below the surface (the skin is at approximately the 6-mm level). With 40 times averaging, the mean clutter and noise in this image is $61-\mathrm{dB}$ below the peak vessel signal. A sequence of $51 \mathrm{~B}$-scans was acquired at $0.254-\mathrm{mm}$ elevational intervals $\left(0.01^{\prime \prime}\right)$ to form a volumetric dataset. Presently, elevational scanning was accomplished by manually translating the array on a linear stage. The elevation resolution defined here as the $-6-\mathrm{dB}$ one-way receive beam waist was measured as $260 \pm 10 \mu \mathrm{m}$. Figure 2(d) shows a 3-D surface rendering of the photoacoustic data compared with a photograph of the underside of the excised skin [Fig. 2(e)]. By eye, these vessels were not visible from the skin surface. The largest vessel diameter as measured in the photograph was approximately $300 \mu \mathrm{m}$.

Maximum data acquisition rate to form a single B-scan is presently limited by the $10-\mathrm{Hz}$ laser pulse-repetition rate and hardware communication ( $\sim 2 \mathrm{sec}$ per B-scan). Future work will include the development of highly parallel data acquisition electronics. This, along with higher pulse-repetition rate lasers, should allow real-time imaging capabilities. Present imaging depths are largely limited by light delivery. With improved light delivery, imaging to greater depths should be likely. The present dynamic-receive focusing capabilities of our system offer a significant advantage over single-element mechanically scanned systems, which have a much smaller, predefined depth of field.
High-frequency photoacoustic imaging with arrays should enable visualization of key features of microvascular function and pathology. This technology may also be used to study microvessel constriction and dilation in response to physiological stimuli. Multiple optical wavelength approaches will enable assessment of microvascular oxygenation. ${ }^{10}$ It should be of significant interest to basic scientists studying angiogenesis in cancer, as well as clinicians such as dermatologists for assessing early stages of peripheral microvascular disease.

In summary, photoacoustic microscopy with highfrequency arrays should provide unique opportunities for clinical and biological impact, and the promising data in this letter should motivate future developments.

\section{Acknowledgment}

This project was sponsored by NIH grants R01 EB000712 and R01 NS46214.

\section{References}

1. P. Carmeliet, "Angiogenesis in life, disease and medicine," Nature (London) 438, 932-936 (2005).

2. K. Maslov, G. Stoica, and L. Wang, "In vivo dark-field reflectionmode photoacoustic microscopy," Opt. Lett., 30(6), 625-627 (2005).

3. H. F. Zhang, K. Maslov, G. Stoica, and L. H. Wang, "Functional photoacoustic microscopy for high-resolution and noninvasive in vivo imaging," Nat. Biotechnol. 24, 848-851 (2006).

4. J. J. Niederhauser, M. Jaeger, R. Lemor, P. Weber, and M. Frenz, "Combined ultrasound and optoacoustic system for real-time highcontrast vascular imaging in vivo," IEEE Trans. Med. Imaging 24(4), 436-440 (2005)

5. D. E. Kruse and K. W. Ferrara, "A new high resolution color flow system using an eigendecomposition-based adaptive filter for clutter rejection," IEEE Trans. Ultrason. Ferroelectr. Freq. Control 49(12), 1739-1754 (2002)

6. D. E. Goertz, D. A. Christopher, J. L. Yu, R. S. Kerbel, P. N. Burns, and F. S. Foster, "High frequency color flow imaging of the microcirculation," Ultrasound Med. Biol. 26, 6371 (2000).

7. T. A. Ritter, T. R. Shrout, R. Tutwiler, and K. K. Shung, "A 30-MHz piezo-composite ultrasound array for medical imaging applications," IEEE Trans. Ultrason. Ferroelectr. Freq. Control 49(2), 217-230 (2002).

8. K. A. Snook, J. Z. Zhao, C. H. Alves, J. M. Cannata, W. H. Chen, R. J. Meyer, T. A. Ritter, K. K. Shung, and M. F. Insana, "High frequency transducers for medical ultrasonic imaging," Proc. SPIE 3982, 92-99 (2000).

9. M. L. Li, H. F. Zhang, K. Maslov, G. Stoica, and L. H. V. Wang, "Improved in vivo photoacoustic microscopy based on a virtualdetector concept," Opt. Lett. 31(4), 474-476 (2006).

10. K. Maslov, M. Sivaramakrishnan, H. F. Zhang, G. Stoica, and L. V. Wang, "Technical considerations in quantitative blood oxygenation measurement using photoacoustic microscopy in vivo," Proc. SPIE 6086, R1-11 (2006). 